\title{
Magnetic Bistability and Nucleation of Magnetic Bubbles in a Layered 2D Organic-Based Magnet $\left[\mathrm{Fe}(\mathrm{TCNE})(\mathrm{NCMe})_{2}\right]\left[\mathrm{FeCl}_{4}\right]$
}

\author{
Jung-Woo Yoo, ${ }^{1}$ V. N. Prigodin, ${ }^{1}$ W. W. Shum, ${ }^{2}$ K. I. Pokhodnya, ${ }^{1,2}$ Joel S. Miller, ${ }^{2}$ and A. J. Epstein ${ }^{1,3}$ \\ ${ }^{1}$ Department of Physics, The Ohio State University, Columbus, Ohio 43210-1117, USA \\ ${ }^{2}$ Department of Chemistry, University of Utah, Salt Lake City, Utah 84112-0850, USA \\ ${ }^{3}$ Department of Chemistry, The Ohio State University, Columbus, Ohio 43210-1173, USA
}

(Received 9 May 2008; published 6 November 2008)

\begin{abstract}
The 2D layered organic-based magnet $\left[\mathrm{Fe}(\mathrm{TCNE})(\mathrm{NCMe})_{2}\right]\left[\mathrm{FeCl}_{4}\right](\mathrm{TCNE}=$ tetracyanoethylene $)$ exhibits a unique macroscopic magnetic bistability between the field-cooled and zero-field-cooled states, which cannot be explained by either superparamagnetic behavior or spin freezing due to spin glass order. This magnetic bistability is described through consideration of the ensemble of uncoupled 2D Ising layers and their magnetization reversal initiated by a field-induced nucleation of magnetic bubbles in individual layers. The bubble nucleation rate strongly depends on the external field and temperature resulting in anomalous magnetic relaxation.
\end{abstract}

PACS numbers: 75.50.Xx, 75.60.Jk, 75.70.Ak, 75.70.Kw

Recent years have witnessed growing attention to organic-based magnets due to their new phenomena and opportunities [1]. The magnetic properties of these materials can be tuned to meet the applications, creating "magnets by design" $[2,3]$. This opens a variety of possibilities for developing materials with the desired magnetic properties, such as magnetic coupling, dimensionality, type of spin, anisotropy, coercivity, etc. One of the interesting phenomena in this class of magnets is "magnetic bistability." Notable examples are spin-crossover complexes, which exhibit thermal transition between high-spin and low-spin states [4], high-spin complexes, which demonstrate macroscopic quantum tunneling of magnetization $[5,6]$ and mixed ferro-ferrimagnetic Prussian blue analogs, which display multiple compensation temperatures [7]. In addition, their magnetic bistabilities often can be controlled by the optical excitation [8-13].

In this Letter, we report unique properties of magnetic bistability of the 2D layered system [Fe(TCNE)$\left.(\mathrm{NCMe})_{2}\right]\left[\mathrm{FeCl}_{4}\right] \quad(\mathrm{TCNE}=$ tetracyanoethylene $) \quad[14]$. The dc magnetization displays anomalous irreversibility between zero-field-cooled (ZFC) and field-cooled (FC) states, which we propose to originate from magnetization reversal of single 2D layers through the nucleation and growth of "bubble domains." We show that the rate of bubble generation together with the bubble size and characteristic relaxation time are strongly contingent on the external magnetic field $(H)$ and thermal energy $\left(k_{B} T\right)$.

Metal-TCNE magnets are a class of organic-based magnets that have been extensively studied. They form a variety of structures ranging from 1-D chain to 3-D network structures, which even show room temperature magnetic ordering for some compositions [15-18]. For example, the $\mathrm{V}(\mathrm{TCNE}) x, x \sim 2$ has a magnetic ordering temperature $\left(T_{c}\right) \sim 400 \mathrm{~K}$ with highly spin-polarized valence and conduction bands $[15,16,19]$. Recently, the first crystal structure of a metal-TCNE magnet, $[\mathrm{Fe}(\mathrm{TCNE})$ $\left.(\mathrm{NCMe})_{2}\right]\left[\mathrm{FeCl}_{4}\right]$, was reported [14]. The structure consists of undulating layers composed of $\mathrm{Fe}^{\mathrm{II}}$ ions with a

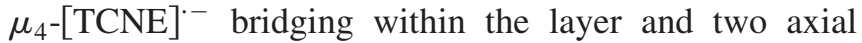
MeCNs coordinations [14]. There are no covalent bonds between layers [14]. Additional paramagnetic $\left[\mathrm{FeCl}_{4}\right]^{-}$ anions occupy sites between the layers of [Fe(TCNE)$\left.(\mathrm{NCMe})_{2}\right]^{+}$but do not contribute to the magnetic ordering [14]. The absence of bridging ligands between the layers suggests only dipolar coupling exists between the layers. In each layer, the magnetic coupling between spin in

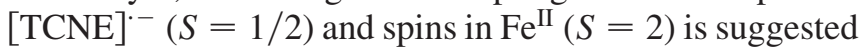
to be antiferromagnetic resulting in ferrimagnetic order [14] similar to other metal-TCNE magnets [15-18]. Thus, each individual layer is considered as an ideal 2D ferrimagnetic Ising system.

The polycrystalline powder samples of [Fe(TCNE)$\left.(\mathrm{NCMe})_{2}\right]\left[\mathrm{FeCl}_{4}\right][14]$ were sealed under vacuum in quartz glass tubes for both static and dynamic susceptibility measurements to protect samples from moisture and oxidation. The grains of the powder sample are composed of aggregated crystallite slabs of stacked 2D layers [20]. The dc magnetic measurements were performed in a Quantum Design MPMS-XL SQUID magnetometer, while dynamic susceptibilities were recorded by a Quantum Design PPMS-9 using the ACMS option.

The FC and ZFC magnetizations upon warming at different external fields are shown in Fig. 1(a). A strong irreversibility is observed between FC and ZFC states [14]. The ZFC magnetization is not only strongly suppressed below the irreversible temperature, $T_{\mathrm{ir}}$, but it is also nearly negligible over a wide temperature range below $T_{c}$ at low field. The ac susceptibility also exhibits the slowing down of spin dynamics as temperature $(T)$ decreases through $T_{c}$, which was attributed to spin glasslike behavior [14]. The strong irreversibility may be attributed 
(a)

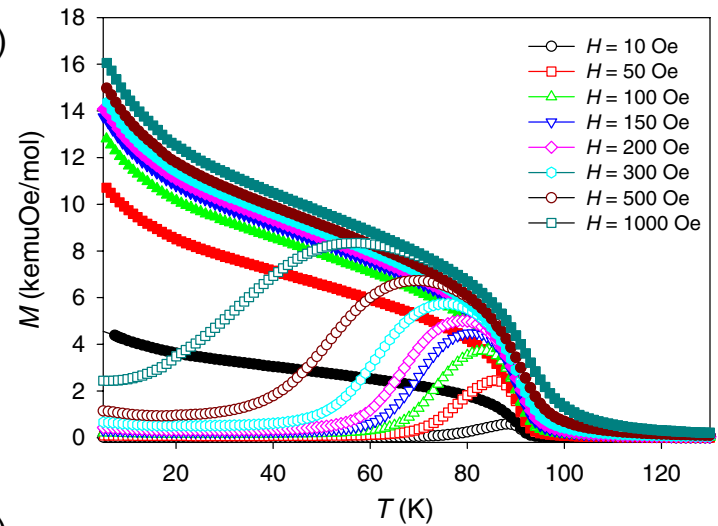

(b)

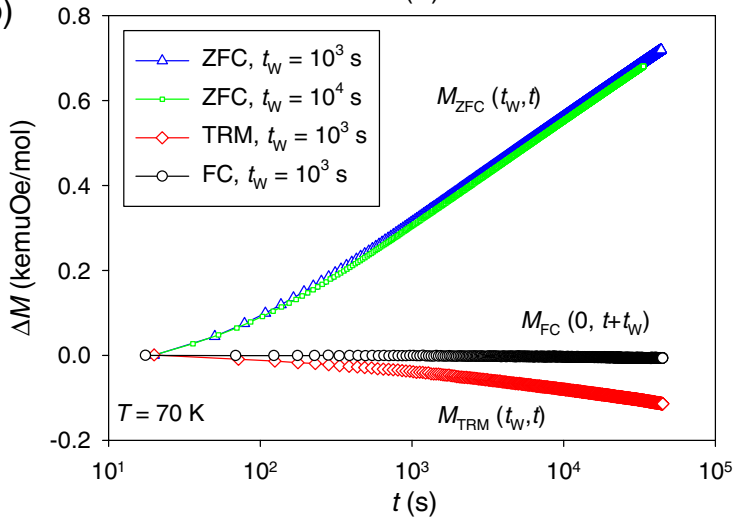

FIG. 1 (color online). (a) Field-cooled (solid symbols) and zero-field-cooled (open symbols) magnetization for different applied magnetic fields $(H=10,50,100,150,200,300,500$, 1000 Oe). (b) Time relaxation of $M_{\mathrm{ZFC}}, M_{\mathrm{FC}}$, and $M_{\mathrm{TRM}}$ at $T=$ $70 \mathrm{~K}, H=100$ Oe for waiting time $t_{w}=10^{3}$ and $10^{4} \mathrm{~s}$.

to (a) a metamagnetic phase transition due to the interlayer coupling, (b) a superparamagnetic blocking energy, (c) a strong spin freezing due to spin glass order, and/or (d) an irreversible magnetic domain formation as the sample is cooled through $T_{c}$ with an applied field. The observed hysteresis in $\left[\mathrm{Fe}(\mathrm{TCNE})(\mathrm{NCMe})_{2}\right]\left[\mathrm{FeCl}_{4}\right]$ features a large coercive field and remanence in contradiction to the behavior of a metamagnet, whose hysteresis curve shows steplike feature due to a first order phase transition [21].

The time dependent relaxation of ZFC, FC, and thermoremanent magnetization (TRM) at $70 \mathrm{~K}$ with an external field 100 Oe is shown in Fig. 1(b). It is well known that the canonical spin glass system exhibits so-called "aging" due to slow growth of the coherence length toward the equilibrium state [22]. The time dependent relaxation of the ZFC magnetization displays no obvious aging effects for waiting times $t_{w}=10^{3} \mathrm{~s}$ and $10^{4} \mathrm{~s}$ [see Fig. 1(b)] indicating absence of spin glass order. In addition, another typical signature of spin glass relaxation, "memory effect" [23], was not observed in this material.

For superparamagnets, there exists a unique response function $f(t)$ for magnetic relaxation at a given temperature, as described $\Delta M(t)=\Delta H f(t)$, where $\Delta H$ is the change of magnetic field at $t=0$ [24]. Such relaxation can be even extended for a cooperative system like a spin glass with an additional input $t_{w}$ due to memory of historical events of the system [24]. One can readily test this fundamental signature by the principle of superposition, as follows $[23,24]$ :

$$
M_{\mathrm{ZFC}}\left(t_{w}, t\right)=M_{\mathrm{FC}}\left(0, t_{w}+t\right)-M_{\mathrm{TRM}}\left(t_{w}, t\right),
$$

where $M_{\mathrm{FC}}, M_{\mathrm{ZFC}}$, and $M_{\mathrm{TRM}}$ are $\mathrm{FC}, \mathrm{ZFC}$, and thermoremanent magnetization, respectively. Figure 1(b) displays a huge difference in the magnetic relaxation between FC and ZFC states. This suggests that the magnetic irreversibility between $M_{\mathrm{FC}}$ and $M_{\mathrm{ZFC}}$ does not originate from either superparamagnetic blocking or spin glass ordering.

Here, we propose that due to the large interlayer separation, the individual layers are magnetically weakly interacting each layer and can be described by the 2D ferrimagnetic Ising plane. Because the polycrystalline powder samples were used for measurement, the easy axis of individual crystallites are randomly oriented. When the sample was cooled in a field below $T_{c}$, all layers have a component of their magnetization directed along the field providing the macroscopic magnetization of $\mathrm{FC}$ phase. However, if the sample is cooled in the absence of a field, each single layer has magnetization randomly either up or down along its own axis resulting in zero total macroscopic magnetization. The magnetization of a single 2D layer is frozen due to large blocking energy below $T_{c}$. Then, the reorientation of magnetization in those layers by a magnetic field will occur through the nucleation of magnetic bubbles [25].

For a 2D ferrimagnetic Ising system, the mean field spontaneous spin polarization is $S=|\mathcal{T}|^{1 / 2}$ below $T_{c}$, where reduced temperature $\mathcal{T}=1-T / T_{c}$. Far below $T_{c}$, all spins align up or down perpendicular to the layer. If the magnetic field occurs opposite to the magnetization of a layer, it initiates the nucleation of bubble domains. The energy of a bubble domain with a radius $n$ (in units of repeat cell) is estimated [26] to be

$$
E_{n} \sim-\mu_{B} H(2 S)\left(\pi n^{2}\right)+(2 \pi n) S^{2} \sqrt{J K},
$$

where the first term is the energy gain due to reorientation of the bubble spin along the field. The second term represents the exchange energy loss in the bubble wall of thickness, $w \sim \sqrt{J / K}$, that depends on exchange constant $(J)$ and anisotropy constant $(K)$. The maximum bubble energy $E_{n}$, which corresponds to the activation energy $E_{b}$, occurs at the critical radius $n=S \sqrt{J K} / 2 \mu_{B} H$ and $E_{b}=\pi S^{3} J K / 2 \mu_{B} H$. Thus, the rate of bubble nucleation determined by the activation energy $E_{b}$ depends on the magnetic field and temperature. Similar description of magnetic reversal with $1 / H$ dependence of energy barrier was extensively studied in metallurgical magnetic systems [25,27].

The crossover field $H_{c}$ derived from the experimental data is presented in Fig. 2(a). Here, the $H_{c}$ for ZFC to FC crossover is the field at which the magnetization starts to increase sublinearly and irreversibly as presented in minor 


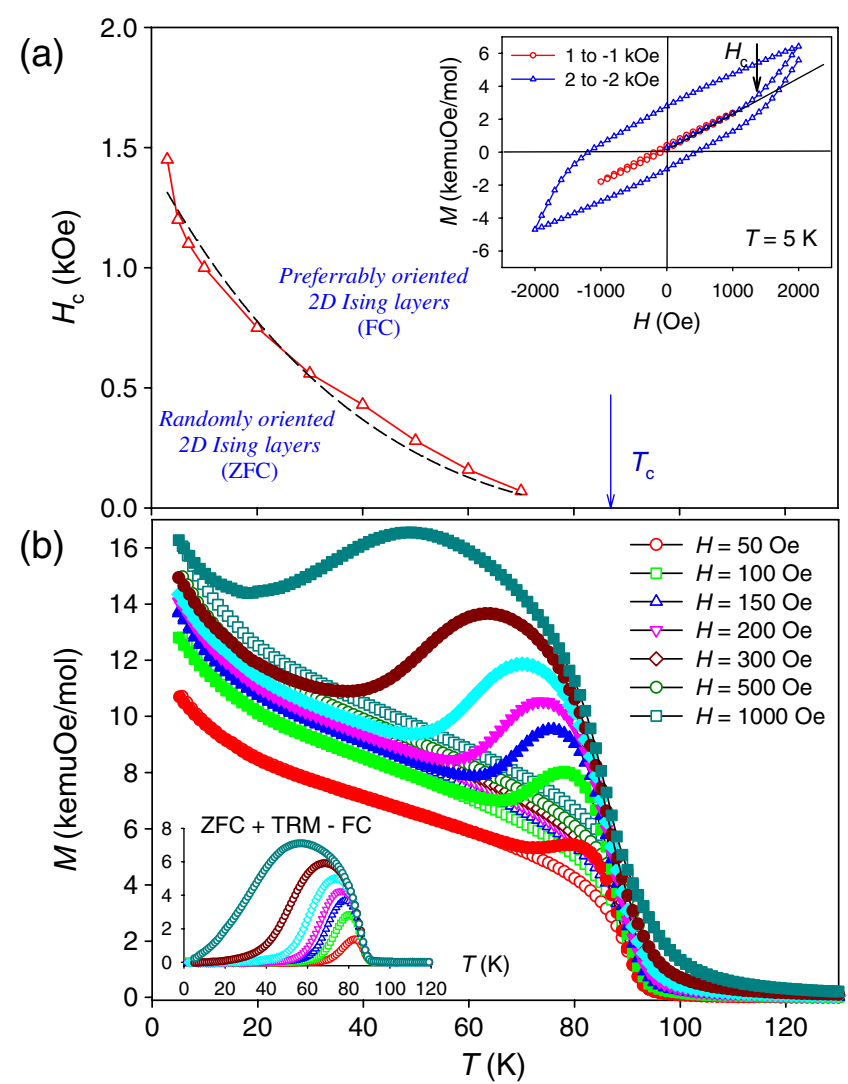

FIG. 2 (color online). (a) A $T$ dependent crossover field obtained from minor hysteresis loop as described in inset. Inset shows minor hysteresis loop for field scan range of \pm 1000 and \pm 2000 Oe. (b) $M_{\mathrm{FC}}(T)$ (open symbols) and $M_{\mathrm{ZFC}}(T)+$ $M_{\mathrm{TRM}}(T)$ (solid symbols) for different applied fields $(H=50$, $100,150,200,300,500,1000$ Oe). Inset shows $M_{\mathrm{ZFC}}(T)+$ $M_{\mathrm{TRM}}(T)-M_{\mathrm{FC}}(T)$ for each applied field.

hysteresis loops in inset of Fig. 2(a). Below $H_{c}, M(H)$ is almost linear with a negligible remanence and coercive field. Above $H_{c}$, the hysteresis loops display irreversibility and remain open upon completing the cycle.

Alternatively, within the bubble nucleation model, $H_{c}$ for the ZFC-FC crossover can be defined at which the bubble nucleation time $\left(\tau=\tau_{0} \exp \left[E_{b} / k_{B} T\right]\right)$ becomes comparable with the measuring time scale $\tau_{m}$. Then, the $T$ dependence of crossover field $H_{c}$ follows

$$
1 / H_{c}=1 / H_{0}+\ln \left(\tau_{m} / \tau_{0}\right) \frac{2 \mu_{B} k_{B} T}{\pi S^{3} J K},
$$

where $H_{0}$ is introduced following the empirical formula of Ref. [27] to limit $H_{c}$ as $T \rightarrow 0$. $H_{0}$ reflects lower limit of bubble size $n \geq w$ [25]. From the above model, $H_{0}$ is estimated as $\mu_{B} H_{0} \sim k_{B} K$. The black dashed line is the fit of $H_{c}(T)$ to Eqn. (3) with employing $\tau_{m} / \tau_{0}=10^{11}$. A quantitative fit yields $H_{0}=1431 \pm 6 \mathrm{Oe}$ and $J K=$ $54.8 \pm 0.7 \mathrm{~K}^{2}$. Taking $J \sim T_{c} \sim 90 \mathrm{~K}$, we get $K \sim$ $0.6 \mathrm{~K}$, which is close to the value $K \sim 0.2 \mathrm{~K}$ estimated from the obtained $H_{0}$. Here, the $K$ values give an order of $\sim 10$ repeat unit cell for the wall thickness as well as minimum size of bubble as $T \rightarrow 0$. A similar $T$ dependence for coercive field due to bubble nucleation was reported in metallurgical magnets [27].

The $M_{\mathrm{ZFC}}(T)+M_{\mathrm{TRM}}(T)$ and $M_{\mathrm{FC}}(T)$ for different applied fields are shown in Fig. 2(b). These data were collected while increasing the $T$ with same rate of linear $T$ sweep, $\delta T / \delta t=0.25 \mathrm{~K} / \mathrm{min}$. There exists large differences between $M_{\mathrm{ZFC}}(T)+M_{\mathrm{TRM}}(T)$ and $M_{\mathrm{FC}}(T)$ over a wide $T$ range that depends on the applied field. We attribute this difference to the thermal activation of bubble domains. If the sample is warmed from ZFC state, the applied field $H$ overcomes $H_{c}$ at certain $T$ initiating nucleation of bubbles. Here, the activation of bubble introduce strong asymmetric magnetic relaxation between $M_{\mathrm{ZFC}}(t)$ and $M_{\mathrm{TRM}}(t)$ as shown in Fig. 2(b).

Below $T_{c}$, the rate of magnetic response to external magnetic field in the ZFC phase also is determined by the nucleation rate. The relaxation time $(\tau)$ is anticipated to behave as $\tau \sim \tau_{0} \exp \left[E_{b} / k_{B} T\right]$. Thus, the relaxation time has the following temperature and field dependencies,
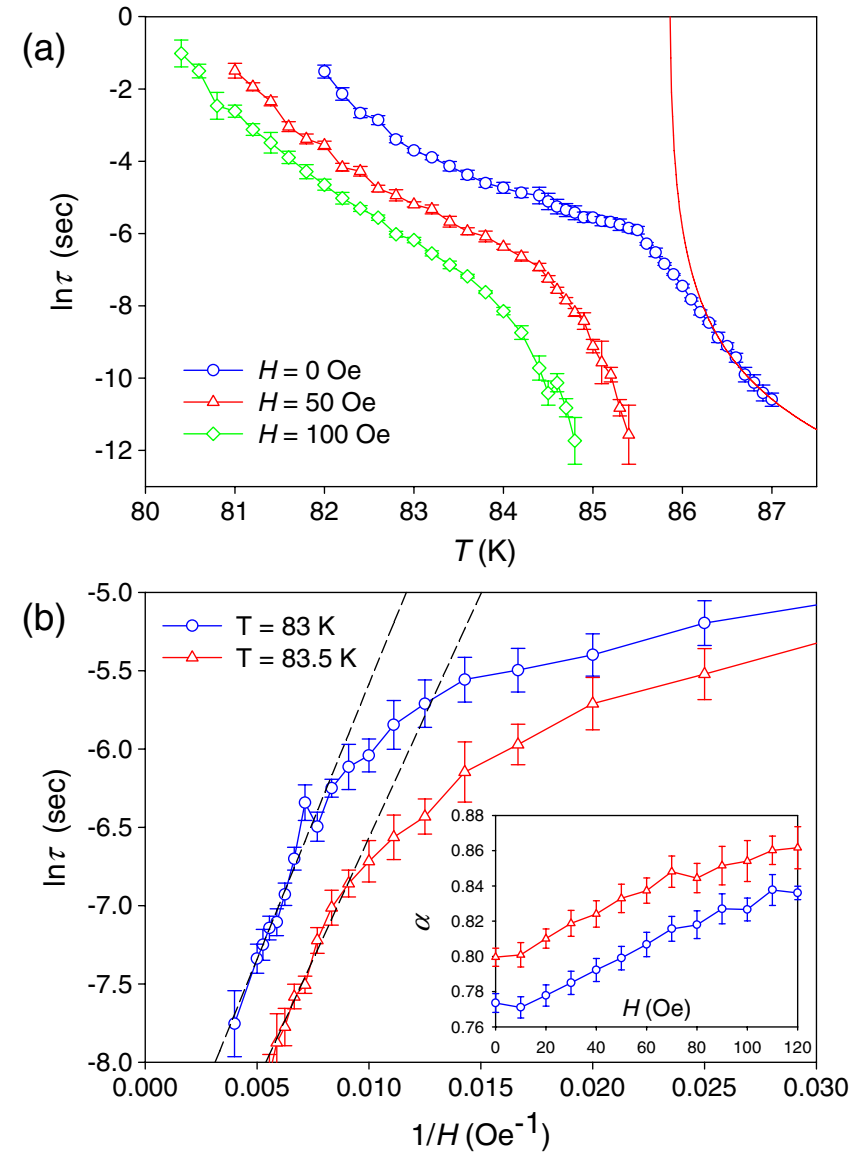

FIG. 3 (color online). (a) The characteristic relaxation time $\tau_{c}$ (T) for dc applied field $H=0,50$, and $100 \mathrm{Oe}$, obtained from complex linear susceptibility. The dashed line indicates a powerlaw fit. (b) The characteristic relaxation time $\tau_{c}(H)$ for a fixed temperature $T=83.0$ and $83.5 \mathrm{~K}$. Inset displays broadening of width of spin relaxation time distribution as increasing external field. 
$\ln \tau \propto|\mathcal{T}|^{3 / 2} / T$ and $\ln \tau \propto 1 / H$. The median relaxation time $\left(\tau_{c}\right)$ together with distribution width of $\tau(\alpha)$ can be determined from the complex linear susceptibility $\left[\chi_{\operatorname{lin}}(\omega)=\chi^{\prime}(\omega)+i \chi^{\prime \prime}(\omega)\right]$ through the Cole-Cole analysis $\left[\chi=\chi_{S}+\frac{\chi_{0}+\chi_{s}}{1+\left(i \omega \tau_{c}\right)^{1-\alpha}}\right.$, where $\chi_{0}$ and $\chi_{s}$ are the isothermal $(\omega=0)$ and adiabatic $(\omega \rightarrow \infty)$ susceptibilities, respectively] [28]. The Cole-Cole analyses were performed following the procedure of Dekker et al. [29].

The temperature dependencies of the characteristic relaxation times for different applied fields $(H=0,50$, $100 \mathrm{Oe}$ ) are displayed in Fig. 3(a). The range of frequencies used for ac field was 11 to $10^{4} \mathrm{~Hz}$ with an amplitude of 5 Oe in order to obtain a distribution of relaxation times. All data were collected with increasing temperature from the ZFC state. As the temperature decreases through the transition, the median relaxation time increases, indicating the growth of the correlation length of the spin system. When there is no applied field, the critical slowing down near $T_{c}\left(T>T_{c}\right)$ is characterized as a power law, $\tau \propto$ $\tau_{0}|\mathcal{T}|^{-z \nu}$, where $\nu$ is the critical exponent for correlation length $\left(\xi \sim \mathcal{T}^{\nu}\right)$ and $z$ is the dynamic critical exponent $\left(\tau \sim \xi^{z}\right)$, respectively. Here, scaling for $z \nu$ varies for a different set of $\tau_{0}$ and $T_{c}$ as well as the temperature fitting range. For a fixed value of $\tau_{0}=10^{-9} \mathrm{~s}$, the highest confident value for $z \nu=2.26 \pm 0.06$ and $T_{c}=85.90 \pm$ $0.05 \mathrm{~K}$ were obtained for the fitting range of $86.2 \mathrm{~K} \leq$ $T \leq 87.0 \mathrm{~K}$ [dashed line in Fig. 3(a)]. The obtained $z \nu$ value is significantly lower than the typical values for spin glass systems [22]. Taking exact value of $\nu=1$ for 2D Ising model [30], the $z$ value is within the range of many theoretical simulations $(2.06 \leq z \leq 2.35)$ for dynamic $2 \mathrm{D}$ Ising model [31]. When the field is applied, a substantial decrease of the overall relaxation times is observed.

The evolution of $\tau_{c}$ of spins with increasing applied field at a fixed temperature $(T=83.0$ and $83.5 \mathrm{~K})$ is displayed in Fig. 3(b). The results exhibit asymptotic behavior of spin relaxation, $\ln \tau \sim 1 / H$ as $1 / H \rightarrow 0$. The dashed lines are fit to the linear region of $\ln \tau$. Here, the slopes correspond to $\pi S^{3} J K /\left(2 k_{B} T \mu_{B}\right)$. At $T=83 \mathrm{~K}$, the slope is 331.7 , which produces $J K=191 \mathrm{~K}^{2}$. The inset in Fig. 3(b) displays $\alpha$ as a function of external field. The monotonic increase of $\alpha$ can also be due to domain activation.

In summary, we reported an unusual macroscopic magnetic bistability of the FC and ZFC magnetization in 2D layered organic-based magnet $\left[\mathrm{Fe}(\mathrm{TCNE})(\mathrm{NCMe})_{2}\right]-$ $\left[\mathrm{FeCl}_{4}\right]$. We propose that this unique magnetic bistability can be explained through consideration of an ensemble of uncoupled 2D Ising layers and their magnetic reversal initiated by the nucleation of bubbles. Here, the molecule-based systems which can be adapted to different situations via organic methodology provide new materials that displays new macro-microscopic phenomena as well as solid state physics of static or dynamic phase transitions.
This work was supported in part by the AFOSR Grant No. FA9550-06-1-0175, DOE Grants No. DE-FG0201ER45931, DE-FG02-86ER45271, and DE-FG0393ER45504.

[1] A. J. Epstein, MRS Bull. 28, 492 (2003).

[2] J. S. Miller and A. J. Epstein, Angew. Chem., Int. Ed. Engl. 33, 385 (1994).

[3] J.S. Miller and A. J. Epstein, Chem. Eng. News 73, 30 (1995).

[4] P. Gütlich and H. Goodwin, Top. Curr. Chem. 233, 1 (2004).

[5] J. R. Friedman, M. P. Sarachik, J. Tejada, and R. Ziolo, Phys. Rev. Lett. 76, 3830 (1996).

[6] L. Thomas et al., Nature (London) 383, 145 (1996).

[7] S. I. Ohkoshi, Y. Abe, A. Fujishima, and K. Hashimoto, Phys. Rev. Lett. 82, 1285 (1999).

[8] O. Sato, T. Iyoda, A. Fujishima, and K. Hashimoto, Science 272, 704 (1996).

[9] D. A. Pejaković, J. L. Manson, J.S. Miller, and A. J. Epstein, Phys. Rev. Lett. 85, 1994 (2000).

[10] D. A. Pejaković, C. Kitamura, J. S. Miller, and A. J. Epstein, Phys. Rev. Lett. 88, 057202 (2002).

[11] S. Ohkoshi et al., J. Am. Chem. Soc. 128, 5320 (2006).

[12] J. W. Yoo et al., Phys. Rev. Lett. 97, 247205 (2006).

[13] J. W. Yoo et al., Phys. Rev. Lett. 99, 157205 (2007).

[14] K. I. Pokhodnya et al., J. Am. Chem. Soc. 128, 15592 (2006).

[15] J. M. Manriquez et al., Science 252, 1415 (1991).

[16] K. I. Pokhodnya, A. J. Epstein, and J. S. Miller, Adv. Mater. 12, 410 (2000).

[17] J.S. Miller and A. J. Epstein, Chem. Commun. (Cambridge) 13, 1319 (1998).

[18] J. Zhang et al., Angew. Chem., Int. Ed. Engl. 37, 657 (1998).

[19] V. N. Prigodin et al., Adv. Mater. 14, 1230 (2002).

[20] See EPAPS Document No. E-PRLTAO-101-002844 for SEM images of $\left[\mathrm{Fe}(\mathrm{TCNE})(\mathrm{NCMe})_{2}\right]\left[\mathrm{FeCl}_{4}\right]$ grains. For more information on EPAPS, see http://www.aip.org/ pubservs/epaps.html.

[21] E. Stryjewski and N. Giordano, Adv. Phys. 26, 487 (1977).

[22] J. A. Mydosh, Spin Glasses: An Experimental Introduction (Taylor and Francis, London, 1993).

[23] R. Mathieu, P. Jönsson, D. N. H. Nam, and P. Nordblad, Phys. Rev. B 63, 092401 (2001).

[24] L. Lundgren, P. Nordblad, and L. Sandlund, Europhys. Lett. 1, 529 (1986).

[25] B. Barbara, Solid State Sci. 7, 668 (2005).

[26] C. Kittel, Rev. Mod. Phys. 21, 541 (1949).

[27] B. Barbara and M. Uehara, IEEE Trans. Magn. 12, 997 (1976).

[28] K. S. Cole and R. H. Cole, J. Chem. Phys. 9, 341 (1941).

[29] C. Dekker et al., Phys. Rev. B 40, 11243 (1989).

[30] A.Z. Patashinskii and V.L. Pokrovskii, in Fluctuation Theory of Phase Transitions (Pergamon, Oxford, 1979).

[31] M. P. Nightingale and H. W. J. Blote, Phys. Rev. Lett. 76, 4548 (1996). 